Supplemental Information

\title{
Kinetics of histidine-tagged protein association to nickel- decorated liposome surfaces
}

Gokul Raghunath, R. Brian Dyer*

Department of Chemistry, Emory University, 1515 Dickey Drive, Atlanta, Georgia 30322, United States

\section{CONTENTS}

Supplementary materials and methods

Supplementary figures and tables

Supplementary references
S2-S4

S5-S19

S20 
Supplementary Materials and Methods.

Protein expression and purification. All proteins used for the study- SfGFP, -30 GFP and EcDHFR were expressed and purified following steps as detailed elsewhere. ${ }^{1}$ Briefly, all genes were expressed by using E. coli strain BL21(DE3) with Luria-Bertani (LB) medium containing $100 \mu \mathrm{g} / \mathrm{mL}$ ampicillin. Care was taken to pick a single ampicillin resistant colony that was then inoculated into a $20 \mathrm{~mL}$ culture flask containing $5 \mathrm{~mL}$ of LB (with ampicillin) medium at $37^{\circ} \mathrm{C}$ overnight inside a shaker incubator maintained at $200 \mathrm{rpm} .1 \mathrm{~mL}$ of the starter culture was then inoculated into a large conical flask containing $1 \mathrm{~L}$ of LB medium with antibiotic and allowed to grow until the optical density at $600 \mathrm{~nm}$ reached 0.6-1.0. Next, inducer was added to a final concentration of $1 \mathrm{mM}$. Either IPTG or L-Arabinose was used as an inducer depending on the construct of interest. The resulting culture was subjected to vigorous shaking in a shaking incubator and allowed to grow for at least 6 hours to overnight. The cells were then harvested by centrifugation and subjected to cell lysis.

The pellets were resuspended in a lysis buffer containing $50 \mathrm{mM}$ Tris, $150 \mathrm{mM} \mathrm{NaCl}, 5 \mathrm{mM} \beta-$ Mercaptoethanol, containing protease inhibitor (from tablets of protease inhibitor cocktail). 1 $\mathrm{mg} / \mathrm{mL}$ of Lysozyme was then added to the mixture, stirred on ice for an hour and sonicated on ice (Sonic Dissemble model 500, Fisher Scientific, Pittsburgh, PA). The lysed cells were then subjected to centrifugation, and the supernatant was filtered and purified using HisPrep affinity column on an AKTA FPLC system (GE Healthcare, Pittsburgh, PA). The proteins were eluted by using an imidazole gradient to competitively unbind the attached proteins to the Ni-NTA column. The eluted protein was subjected to desalting and concentrated using an Amicon concentrator with a $10 \mathrm{kDa}$ molecular weight cutoff. The protein samples were then buffer exchanged to 50 mM MOPS buffer maintained at a pH 7.2. For EcDHFR samples, a small concentration of reducing agent (1 mM DTT) was added to prevent the possibility of disulfide formation. ${ }^{2}$

Isothermal Titration Calorimetry. All ITC experiments reported in the manuscript was performed using a MicroCal VP-ITC in low-feedback mode. A total of 31 injections were performed, resulting in titration of liposomes stock in the titrator syringe into a thoroughly rinsed cell chamber containing the protein sample. A Ni-NTA DGS concentration of $350 \mu \mathrm{M}$ (Total liposome concentration of $1.75 \mathrm{mM}$ in case of $20 \mathrm{~mol} \%$ Ni-NTA DGS) was used in all experiments, and the DOPC concentration was varied to accommodate changing chelator lipid densities. The protein stock solutions were maintained at $9 \mu \mathrm{M}$ unless otherwise reported. The reference power was maintained at $10 \mu \mathrm{Cal} / \mathrm{sec}$ and the cell temperature $\left(20^{\circ} \mathrm{C}\right)$ was equilibrated for at least 30 minutes. The spacing between injections were set 360 seconds and data from the first injection was always discarded due to artifacts from dilution. Data analysis was performed by the MicroCal Analysis software, and the obtained enthalpograms were fit to one set of sites (for sample void of cholesterol) or two set of sites model (for samples containing cholesterol) and the coefficients are reported in Table S2.

Analysis of steady state fluorescence data. Steady state fluorescence quenching from SfGFPliposome interaction was analyzed based on traditional fluorometric assays of protein binding to liposomes. ${ }^{3}$ We employed the use of the Hill equation as described previously. ${ }^{4} \mathrm{~A}$ constant ligand (Ni-NTA DGS) concentration was chosen to eliminate changes to light scattering due to increasing liposome presence and the vesicle samples were incubated with different protein concentrations.

$\frac{\Delta F}{\Delta F_{\max }}=\frac{[P]_{b}}{[P]_{o}}=\frac{1}{1+\left(K_{d} /[P]_{0}\right)^{h}}$

Where $[\mathrm{P}]_{\mathrm{b}},[\mathrm{P}]_{0}$ represents the bound protein concentration and total protein concentrations respectively. $\mathrm{K}_{\mathrm{d}}$ here represents the apparent dissociation constant and $h$ represents the Hill 
coefficient. $\Delta \mathrm{F}$ and $\Delta \mathrm{F}_{\max }$ indicate the fluorescence intensity decrease at a given protein concentration, and the maximal intensity decrease, respectively. Given that the amount of protein bound must correlate with the decrease in fluorescence, we were then able to fit the individual binding isotherms to the Hill equation (Figure S2) to yield apparent dissociation constants that correlate well with the data acquired via ITC. The fit coefficients are summarized in Table S1.

Fluorescence recovery after photobleaching. Lipid lateral mobility changes upon addition of Cholesterol was assessed by using Nikon C2 inverted confocal microscope using an excitation laser centered at $561 \mathrm{~nm}$ and emission captured using a lower energy bandpass filter placed in front of a 3-channel standard Photomultiplier tube detector system. $0.02 \mathrm{~mol} \%$ of Rhodamine PE was doped in to the lipid cakes for FRAP experiments. The lipid cakes were hydrated, extruded and ultimately supported lipid bilayers were formed by spontaneous fusion on plasma cleaned glass surfaces as described elsewhere. ${ }^{5}$ Time-lapse images were acquired over 420 seconds after photobleaching a circular spot of diameters ranging from 10-25 $\mu \mathrm{m}$. The fluorescence recovery transients were subsequently fit to exponential function, enabling us to estimate the lipid lateral mobility and the changes in diffusivity upon changing cholesterol concentration.

Preparation of $\mathrm{Cu}$ and $\mathrm{Co}$ containing liposomes. To change the divalent cations bound to the chelator lipid headgroup, we followed protocols described elsewhere. ${ }^{6}$ Briefly, we doped Ni-free NTA-DGS into DOPC containing lipid cakes at $20 \mathrm{~mol} \%$ density. After liposome extrusion, we incubated the liposome solution in a buffer stock containing 2-fold molar excess of $\mathrm{CuCl}_{2}$ or $\mathrm{CoCl}_{2}$ and the excess divalent cations were removed from solution through several rounds of centrifugal filtration. All liposome samples were subjected to dynamic light scattering measurements and we did not observe any significant changes in the morphology of the liposomes (data not shown), indicating that the addition of divalent cations did not affect the liposomes hydrodynamic diameter. For Co(II) oxidation experiments, we first incubated the Co(II) NTA doped vesicles with SfGFP at varying concentrations for at least 2 hours in room temperature to reach equilibrium. Once the incubation time was complete, we added $10 \mathrm{mM}$ Hydrogen Peroxide to all the samples. ${ }^{7}$ After an hour, we removed the unbound SfGFP from the samples by subjecting them to centrifugal filtration utilizing a $50 \mathrm{kDa}$ molecular weight cutoff filter. After 3 rounds of filtration, we observed little to no presence of SfGFP in the filtrate indicating the successful oxidation of $\mathrm{Co}$ (II) to $\mathrm{Co}$ (III) and subsequently, immobilization of proteins to the surface (data not shown).

Analysis of stopped flow transients. To obtain the rate constants associated with stopped flow transients, we fit the fluorescence decay globally to either bi or tri exponential functions. We noticed that, in presence of cholesterol, protein binding to liposomes exhibited a tri-exponential behavior with no clear concentration dependent trend. While a thorough exploration of these rate constants was beyond the scope of this manuscript, it is certainly possible that long-range structural effects due to protein binding to cholesterol containing liposomes might cause extensive re-arrangement leading to such behavior. ${ }^{8}$ Regardless, we report the rate constants of the slower phase in supplementary figure S10 for reference.

To fit the concentration dependence of slow phase rate constants, we fit the rate constants to a modified sigmoidal fit as shown in equation S2. The fits were performed purely to guide the readers eye, and not to imply any direct physical meaning.

$y=$ base $+\left(\frac{\max }{1+e^{(\text {xhalf }-x) / \text { rate }}}\right)+(a \cdot x)+b$ 
Where base is the baseline, max represents the maximum value, xhalf represents the midpoint of the sigmoidal fit, rate represents the rising rate, a represents the slope of the line (negative slope at higher SfGFP concentrations) and $b$ represents the fitting constant.

Anisotropy correction. To account for the artificial depolarization in anisotropy by light scattering in a turbid medium, we performed a correction, by accounting for the optical density of the solution in the 96-well plate. We slightly modified the protocol followed by Lentz et al. ${ }^{9}$ Briefly, to account for the turbidity of the medium, we utilized liposomes void of the chelator lipid. Given that the change in chelator lipid density was accomplished by varying the total DOPC content, we established a standard curve measurement by keeping the SfGFP concentration the same, and by changing the liposome concentration across a wide range. This changes the optical density of the medium significantly (data not shown). By sampling a wide range of liposome concentration, we were able to obtain a correction factor by fitting the anisotropy depolarization as a function of increasing concentrations to a linear function by using equation (S3)

$r_{o b s}=r^{\prime}-\left(r^{\prime} \cdot K \cdot[D O P C]\right)$

Where robs is the observed anisotropy, r' is the extrapolated anisotropy at zero optical density and $\mathrm{K}$ is the proportionality constant. This correction was used to account for scattering induced depolarization of the polarized light for all data presented in Figure 6C.

Fluorescence Lifetime Measurements. To verify that homo-FRET affects only the fluorescence anisotropy, and not the fluorescence intensity and lifetimes, we measured the change in fluorescence lifetimes of SfGFP emission upon binding to the liposome surface. Liposomes void of quencher lipids were used for all lifetime measurements. All experiments were performed on a Nikon Ti Eclipse Inverted Confocal microscope (C2Si). The confocal system was attached to a Picoquant (Berlin, Germany) Laser Scanning Microscope Time Correlated Single Photon Counting upgrade. For excitation, a $485 \mathrm{~nm}$ pulsed laser was operated at $20 \mathrm{MHz}$ and the fluorescence emission was monitored by utilizing a bandpass filter centered at $520 \mathrm{~nm}$. The emitted fluorescence lifetime traces were analyzed using SymPhoTime 64 software, utilizing Fast FLIM algorithm. All decay traces were fit to bi-exponential functions resulting in two characteristic lifetimes of $1.5 \mathrm{~ns}(\sim 15 \%$ amplitude) and $2.5 \mathrm{~ns}(\sim 85 \%$ amplitude) respectively. It is likely that the two lifetimes are representative of the I form and the B form of the chromophore. ${ }^{10}$ While they are slightly lower than expected, these values are comparable to those obtained in the literature (2.1ns for I-form and $3 \mathrm{~ns}$ for B-form respectively). The instrument response function (IRF) was collected using heavily quenched samples made of Erythrosine B in a saturated solution of Potassium lodide. Concentration dependent changes of fluorescence lifetimes were acquired by using a clear, untreated 96-well plate. 


\section{Supplemental Figures:}

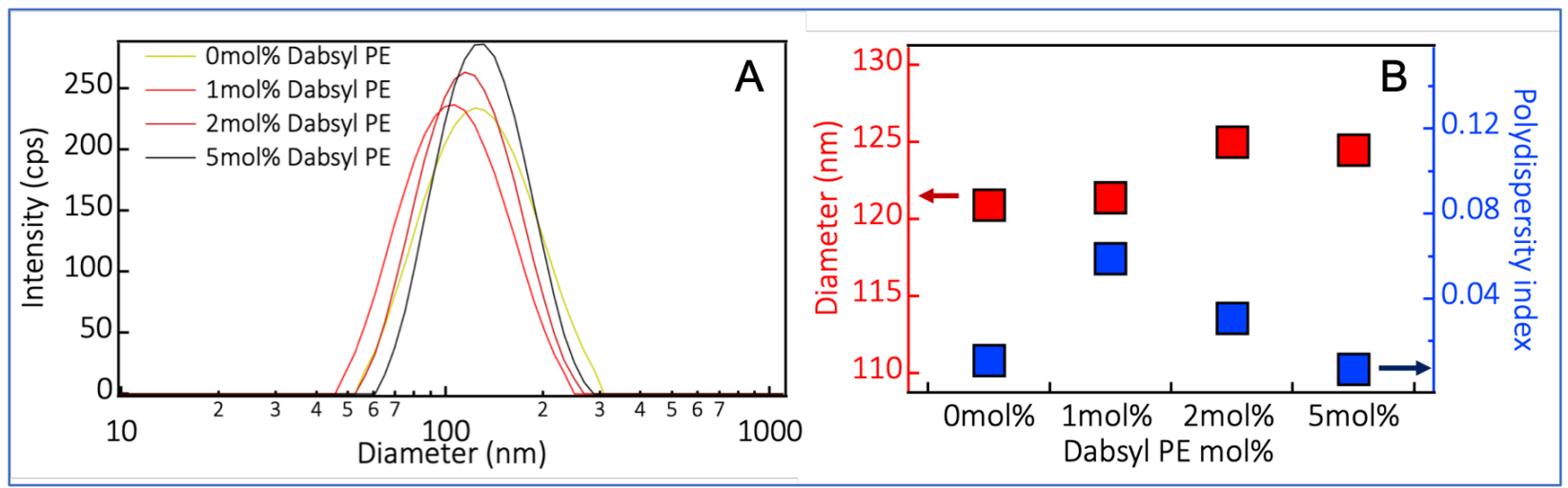

Figure S1. Ensuring proper formation of liposomes with increasing concentrations of Dabsyl PE. (A) Dynamic Light Scattering intensity profile of $1 \mathrm{mM}$ DOPC liposomes containing 0, 1, 2, 5 mol\% Dabsyl PE. (B) Average diameters (Red squares- reported on the axis on the left) and polydispersity index of the liposomes (Blue squares- reported on the axis on the right) of the vesicle samples with increasing Dabsyl PE loading levels. The vesicles were extruded through a $100 \mathrm{~nm}$ polycarbonate membrane as described in the experimental section, leading to a highly monodisperse population. 

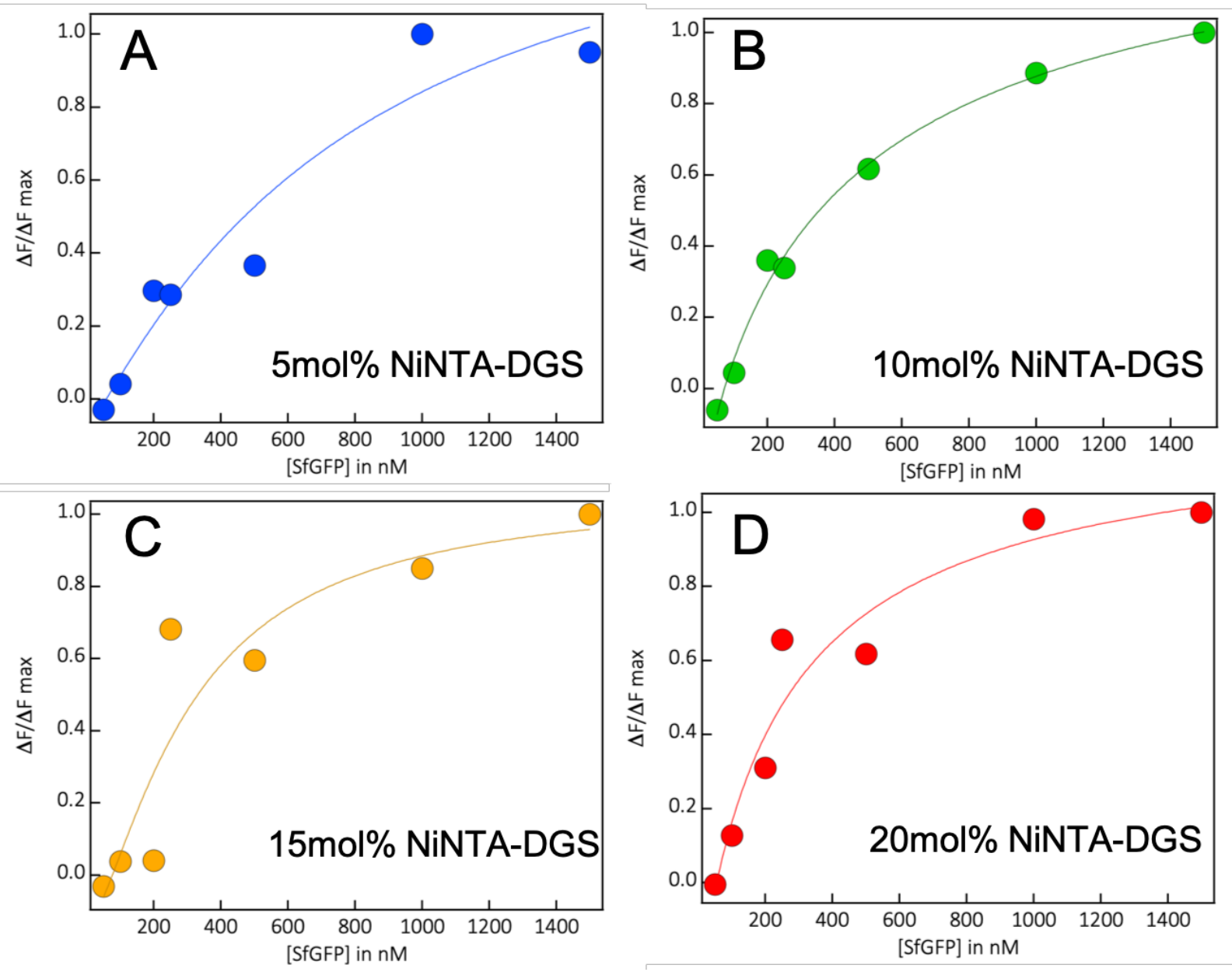

Figure S2. Steady state fluorescence binding isotherms. Change in steady state fluorescence intensity as a function of increasing SfGFP concentration and associated fits to the Hill equation ( $h$ constrained to 1 ). Increasing the chelator lipid density enhances the apparent dissociation constants as reported in Table S1. (A), (B), (C), (D) represent data acquired on liposomes containing 5, 10, 15 and 20 mol\% Ni-NTA DGS respectively.

Table S1. Coefficients from fits in Figure S2.

\begin{tabular}{|c|c|c|c|c|}
\hline Chelator density & $5 \mathrm{~mol} \%$ & $10 \mathrm{~mol} \%$ & $15 \mathrm{~mol} \%$ & $20 \mathrm{~mol} \%$ \\
\hline $\begin{array}{c}\text { Apparent } \\
\text { Dissociation } \\
\text { constant } \mathrm{K}_{\mathrm{d}} \text { (in nM) }\end{array}$ & 856.82 & 379.91 & 351.01 & 247.89 \\
\hline
\end{tabular}




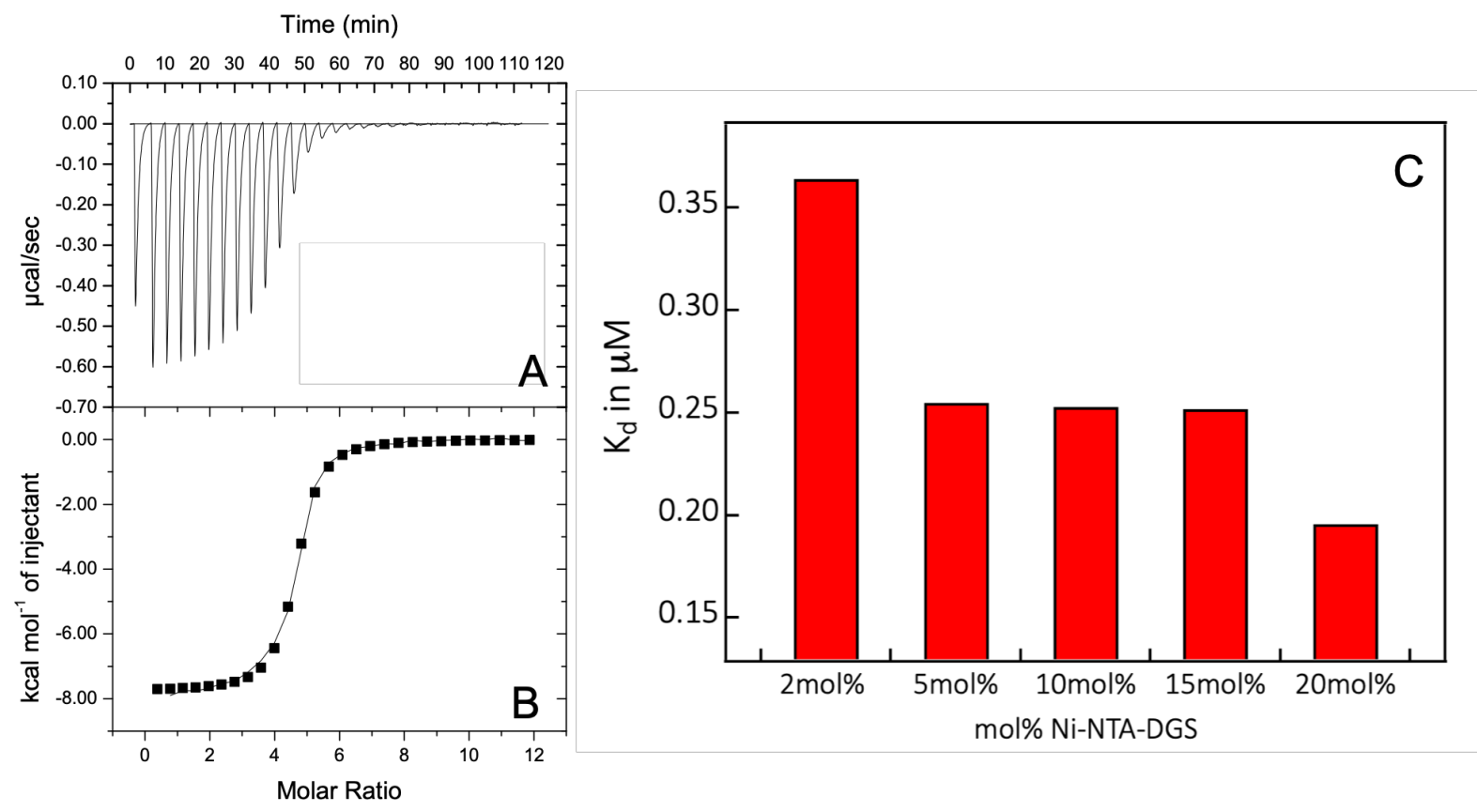

Figure S3. Isothermal titration calorimetry of SfGFP binding to Ni-NTA DGS containing liposomes. (A) Representative ITC thermograms obtained by injecting (350 $\mu$ M Ni-NTA DGS liposomes at a 20 mol\% density) into the cell chamber containing $9 \mu \mathrm{M}$ SfGFP. (B) Enthalpograms obtained under the same conditions. The solid line represents the associated fit to a single set of sites model. (C) Dissociation constants obtained via ITC as a function of increasing chelator lipid densities. 

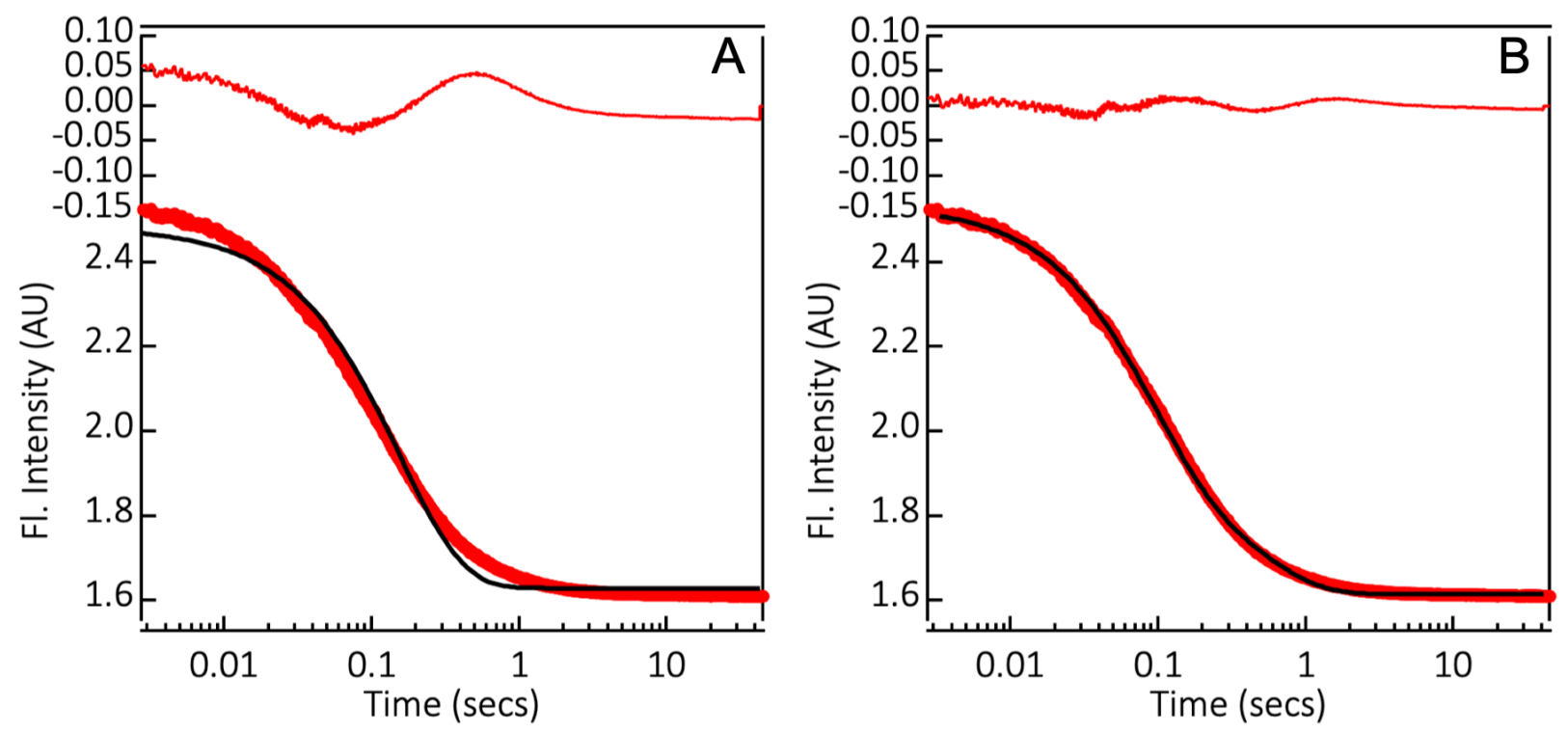

Figure S4. Stopped flow transients and their associated fits Representative stopped flow fluorescence plotting the decrease in fluorescence intensity as a function of time. $200 \mu \mathrm{M}$ Ni-NTA DGS and $1 \mu \mathrm{M}$ SfGFP stock solutions were mixed in an observation chamber. (A) Stopped flow transient and the associated mono-exponential fit. The residuals reported at the top clearly shows that a single exponential is not adequate to describe the fluorescence quenching. (B) Stopped flow transient and associated bi-exponential fit. The residuals reported at the top show that a biexponential fit is appropriate for analysis. 

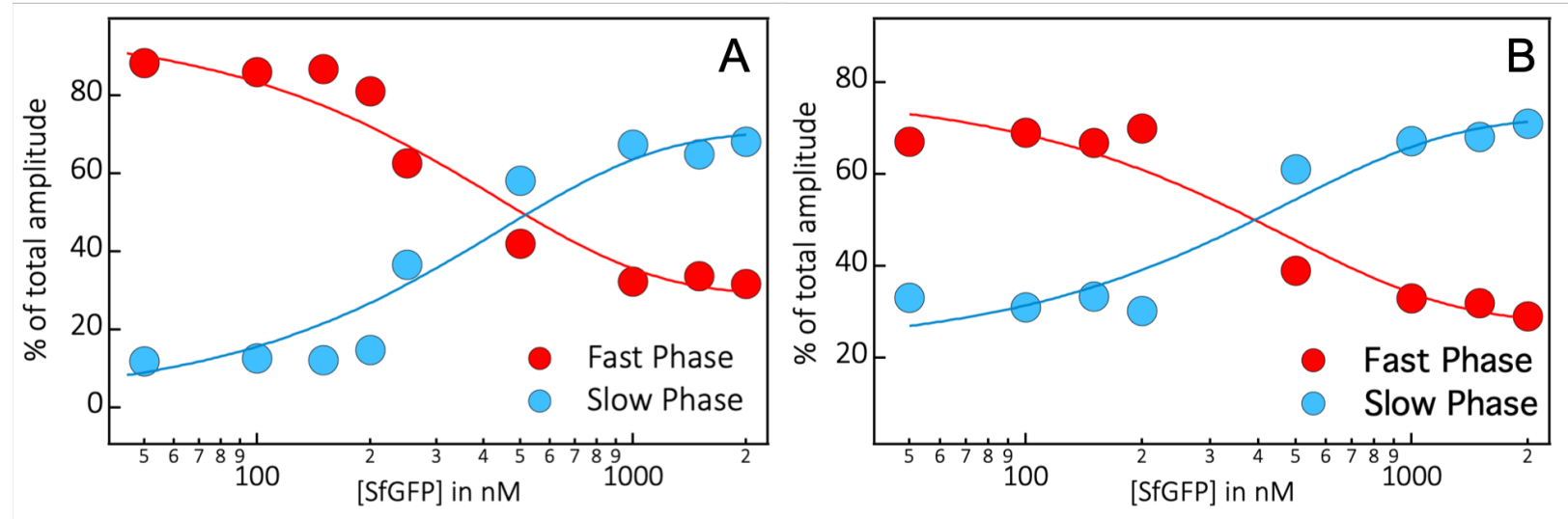

Figure S5. Relative amplitudes of the two exponential phases obtained from stopped flow experiments. (A) represents the relative amplitudes of the fast and the slow phases, for liposomes containing $5 \mathrm{~mol} \%$ Ni-NTA DGS and (B) represents the relative amplitudes of the fast and the slow phases for liposomes containing $20 \mathrm{~mol} \%$ Ni-NTA DGS. As evidenced from the opposing trends, the slow phase amplitudes clearly have a bigger contribution to the overall fluorescence decay relative to the fast phase, particularly at higher protein concentrations. The fast and the slow phase amplitudes were fit to an exponential function to lead the reader's eye and not to imply any direct physical meaning. 

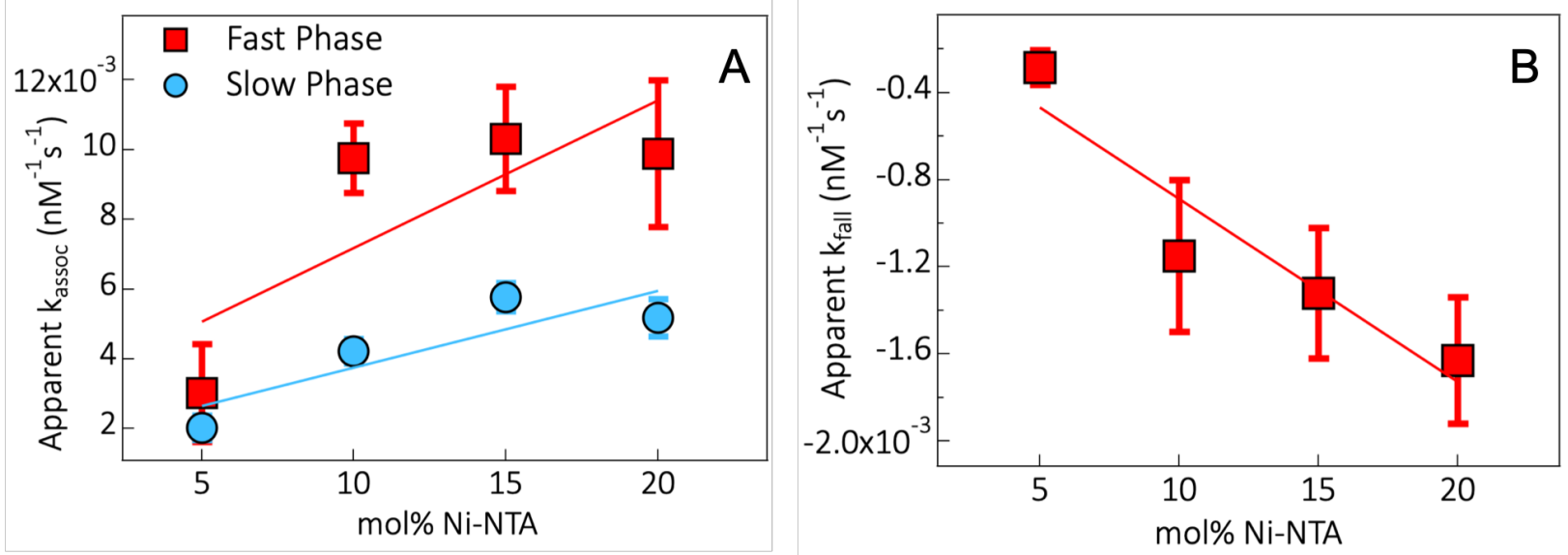

Figure S6. Calculation of apparent association constants. (A) Calculation of apparent association constants from stopped flow fluorescence data. Bi-exponential rate constants $\left(\mathrm{k}_{\text {fast }}\right.$ and $\mathrm{k}_{\text {slow }}$ ) at low concentration $(<500 \mathrm{nM}$ ) data from SfGFP binding to liposomes were fit to a linear function to obtain apparent $\mathrm{k}_{\text {assoc }}\left(\mathrm{nM}^{-1} \mathrm{~s}^{-1}\right)$. The apparent association constants were found to increase as a function of increasing chelator lipid density, indicating a bimolecular behavior for protein binding to liposomes under non-crowding conditions. (B) The slow-phase rates undergo a break in bimolecular behavior to exhibit a negative concentration dependence ([SfGFP] > 500 $\mathrm{nM}$ ). The magnitude of this effect was reported by the use of $k_{\text {fall }}$, a parameter obtained by fitting the rate constant data for the slow-phases at higher SfGFP concentrations, to a linear function. The negative rates are meant to be a qualitative guide and do not imply any direct physical meaning. 

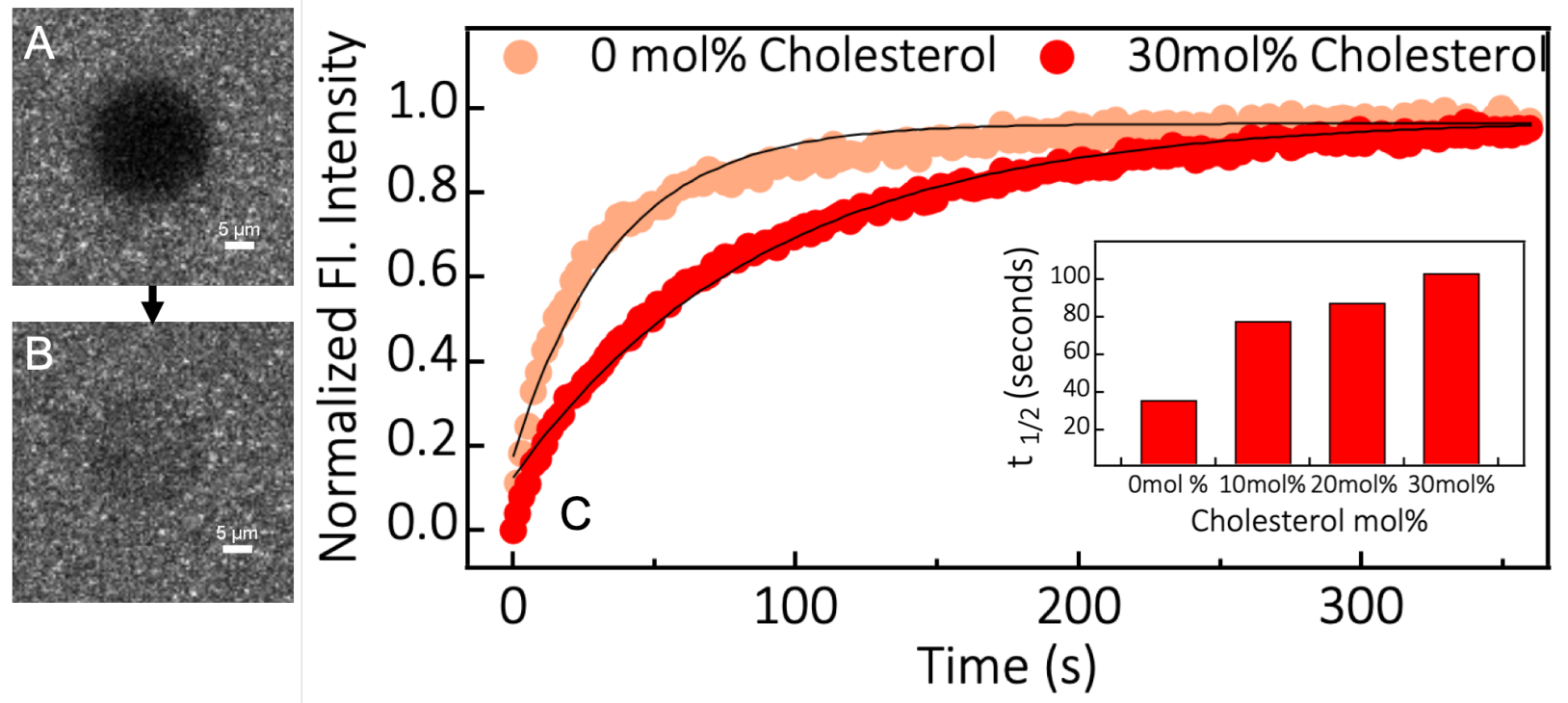

Figure S7. Fluorescence recovery after photobleaching. (A) Representative confocal timelapse image at $t=0$, after photobleaching a spot at high laser powers. (B) Representative confocal image at $t=300$ after recovery of the photobleached sample under observation conditions. Both images were captured by excitation at $561 \mathrm{~nm}$ wavelength, and observation via a bandpass filter centered at $582 \mathrm{~nm} .0 .02 \mathrm{~mol} \%$ of Rhodamine PE was doped into the vesicle composition, and used as a fluorescent reporter. (C) Normalized fluorescence intensity plotted as a function of time representing the recovery of fluorescence after photobleaching. Clearly, increasing cholesterol concentration decreases the lateral mobility of the lipids involved in the formation of the Supported lipid bilayer, indicating the restriction of lateral mobility by cholesterol loading. The recovery kinetics were fit to a single exponential leading to the calculation of a $t_{1 / 2}$ (secs) and reported in the inset. 

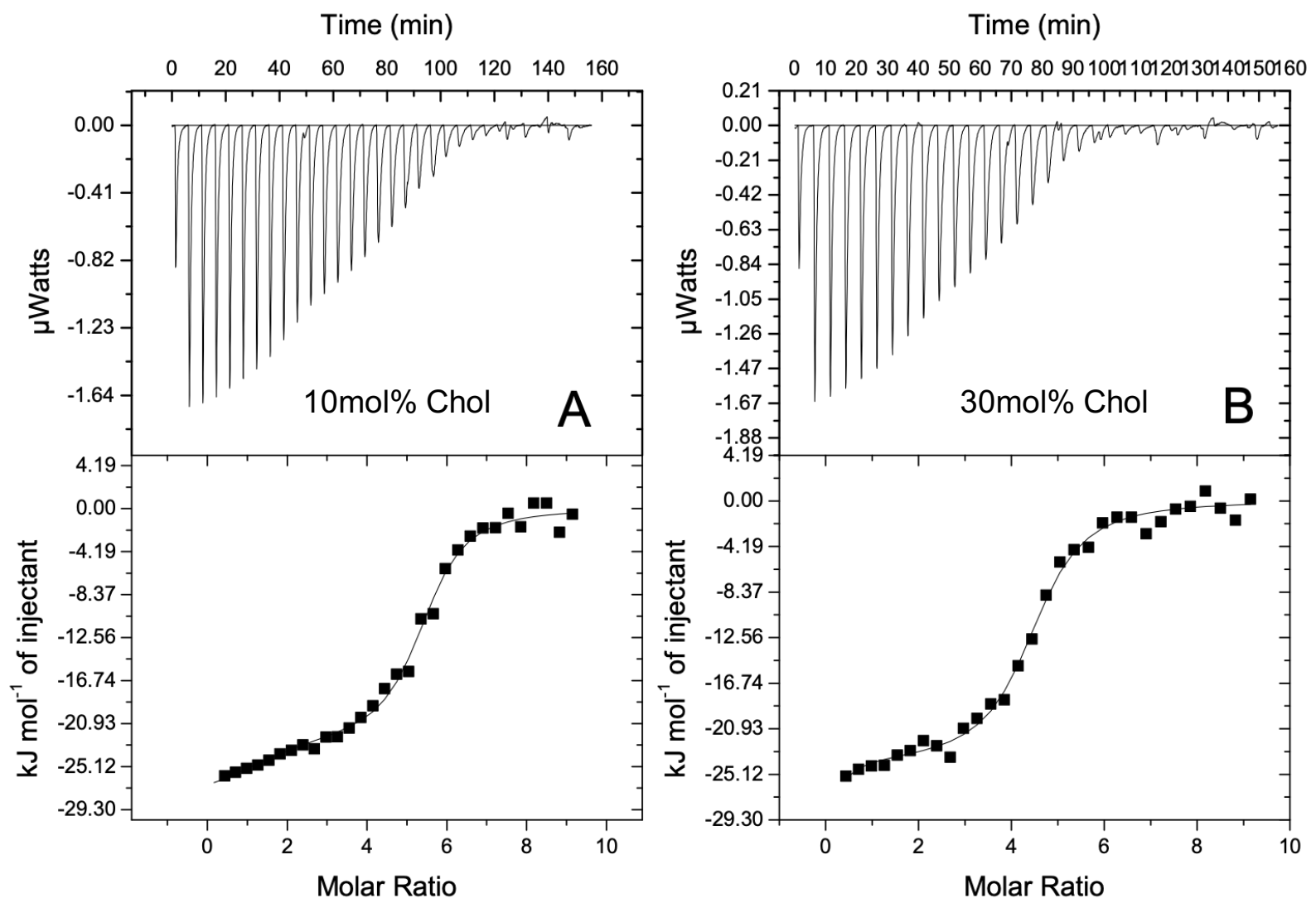

Figure S8. Isothermal titration calorimetry of SfGFP binding to Ni-NTA DGS containing liposomes with varying Cholesterol doping levels. (A) Representative ITC thermograms obtained by injecting $350 \mu \mathrm{M}$ Ni-NTA DGS decorated liposomes (10 mol\% Cholesterol along with $20 \mathrm{~mol} \%$ Ni-NTA DGS) into the cell chamber containing $9 \mu \mathrm{M}$ SfGFP. Enthalpogram obtained under the same conditions is reported in the lower half of the graph. (B) Representative ITC thermograms obtained by injecting $350 \mu \mathrm{M}$ Ni-NTA DGS decorated liposomes $(30 \mathrm{~mol} \%$ Cholesterol along with $20 \mathrm{~mol} \%$ Ni-NTA DGS) into the cell chamber containing $9 \mu \mathrm{M}$ SfGFP. Enthalpogram obtained under the same conditions are reported in the lower half of the graph. Both enthalpograms were fit to a twoset of binding sites model and the dissociation constants and stoichiometries are listed in Table S2 below.

Table S2. Dissociation constants and stoichiometries obtained from fits in Figure S8.

\begin{tabular}{|l|l|l|l|}
\hline Cholesterol density & $10 \mathrm{~mol} \%$ & $20 \mathrm{~mol} \%$ & $30 \mathrm{~mol} \%$ \\
\hline $\mathrm{K}_{\mathrm{d} 1}$ in $(\mathrm{nM})$ & $100 \mathrm{nM}$ & $71.32 \mathrm{nM}$ & $63.29 \mathrm{nM}$ \\
\hline $\mathrm{K}_{\mathrm{d} 2}$ in $(\mathrm{nM})$ & $740 \mathrm{nM}$ & $628 \mathrm{nM}$ & $591 \mathrm{nM}$ \\
\hline Stoichiometry $\left(\mathrm{n}_{1}\right)$ & 1.22 & 1.30 & 1.24 \\
\hline Stoichiometry $\left(\mathrm{n}_{2}\right)$ & 4.40 & 4.04 & 4.24 \\
\hline
\end{tabular}



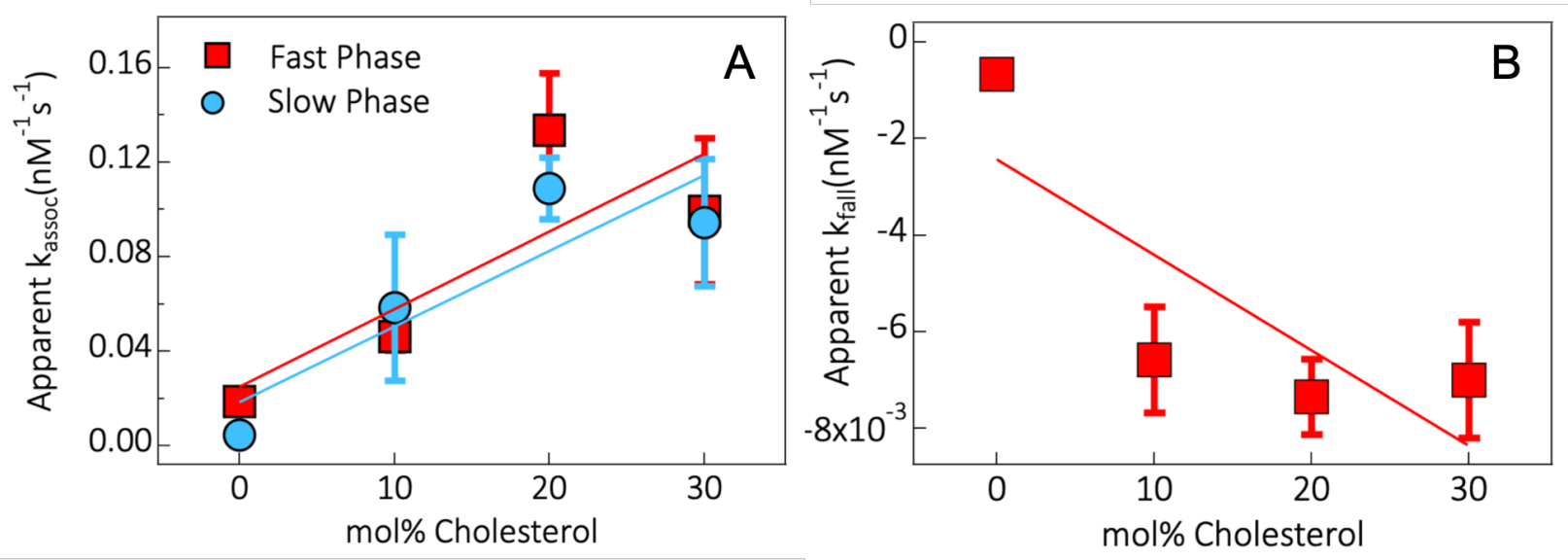

Figure 59. Calculation of apparent association constants. (A) Calculation of apparent association constants from stopped flow fluorescence data. Rate constants from poly exponential fits ( $k_{\text {fast }}$ and $k_{\text {slow }}$ ) at low concentration $(<500 \mathrm{nM})$ data from SfGFP binding to liposomes were fit to a linear function to obtain apparent $k_{\text {assoc }}\left(\mathrm{nM}^{-1} \mathrm{~s}^{-1}\right)$. (B) The slow-phase rates undergo a break in bimolecular behavior to exhibit a negative concentration dependence ([SfGFP] > $500 \mathrm{nM}$ ). The magnitude of this effect was reported by the use of $k_{\text {fall }}$, a parameter obtained by fitting the rate constant data for the slow-phases at higher SfGFP concentrations, to a linear function. The negative rates are meant to be a qualitative guide and do not imply any direct physical meaning. 


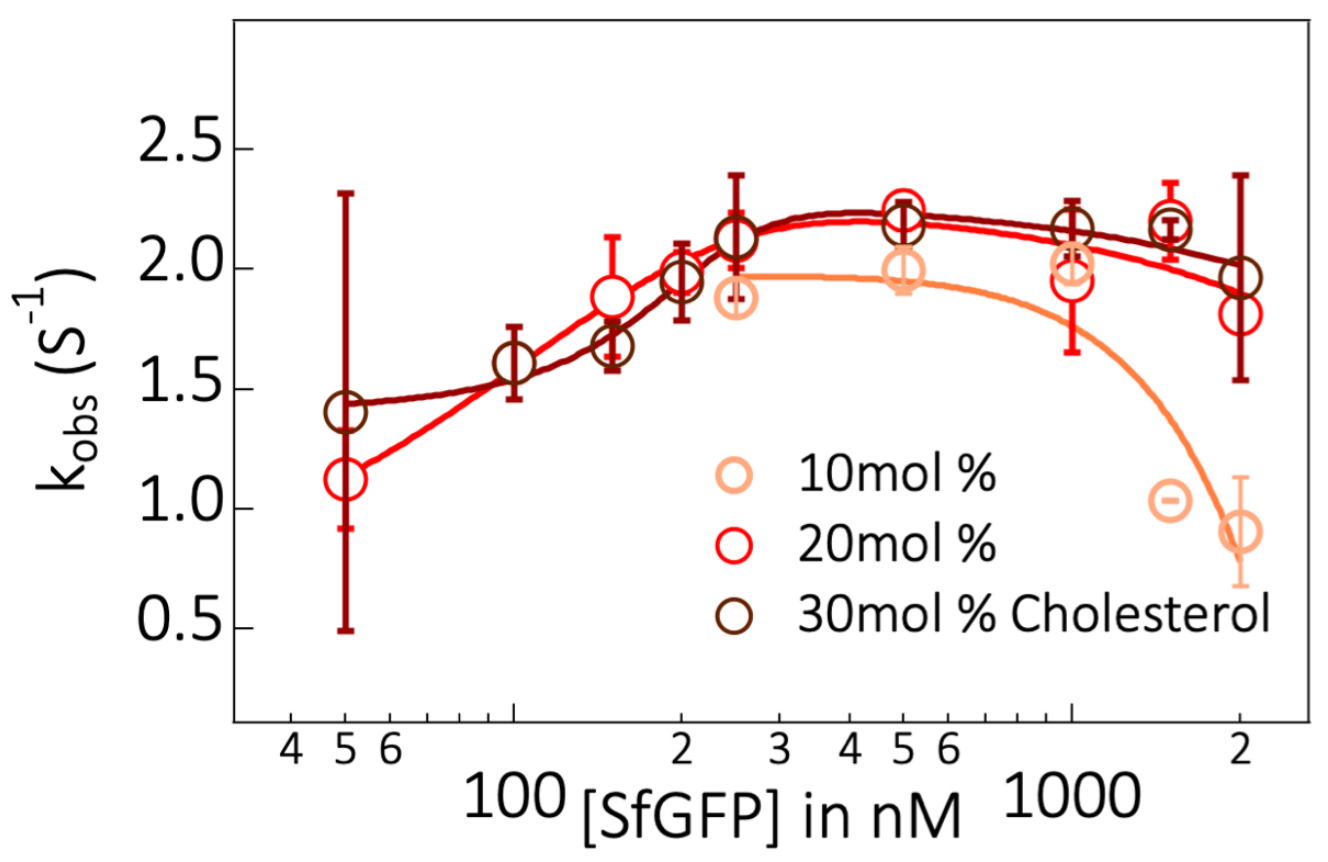

Figure S10. Third phase rate constants observed in stopped flow transients with liposomes containing cholesterol. Concentration dependence of the slower, third phase rates obtained from stopped flow transients and their tri-exponential fits. At 10 mol\% cholesterol doping levels, we didn't observe a tri-exponential at SfGFP concentrations below $250 \mathrm{nM}$. However, a tri-exponential fitting function was necessary to describe the fluorescence decay at higher cholesterol loading levels. 

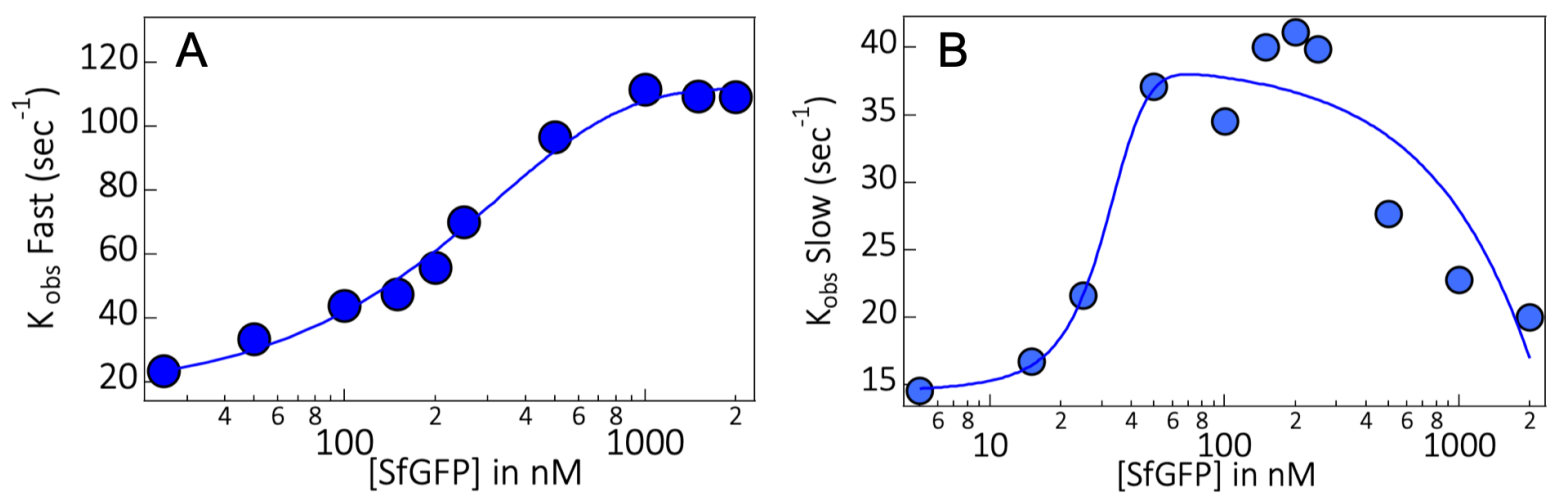

Figure S11. Effects of changing divalent cations on the chelator lipid headgroup. Rate constants from bi-exponential fits to the stopped flow transients. Upon replacing the divalent cation from $\mathrm{Ni}^{2+}$ to $\mathrm{Cu}^{2+}$, we observe a large enhancement in binding rates for both the fast and the slow phases. (A) Fast phase rate constants plotted as a function of SfGFP concentration and (B) Slow Phase rate constants plotted as a function of SfGFP concentration. Despite enhancement in overall binding rates, we continued to observe the trend of decreasing rate constants in the slow phase. 

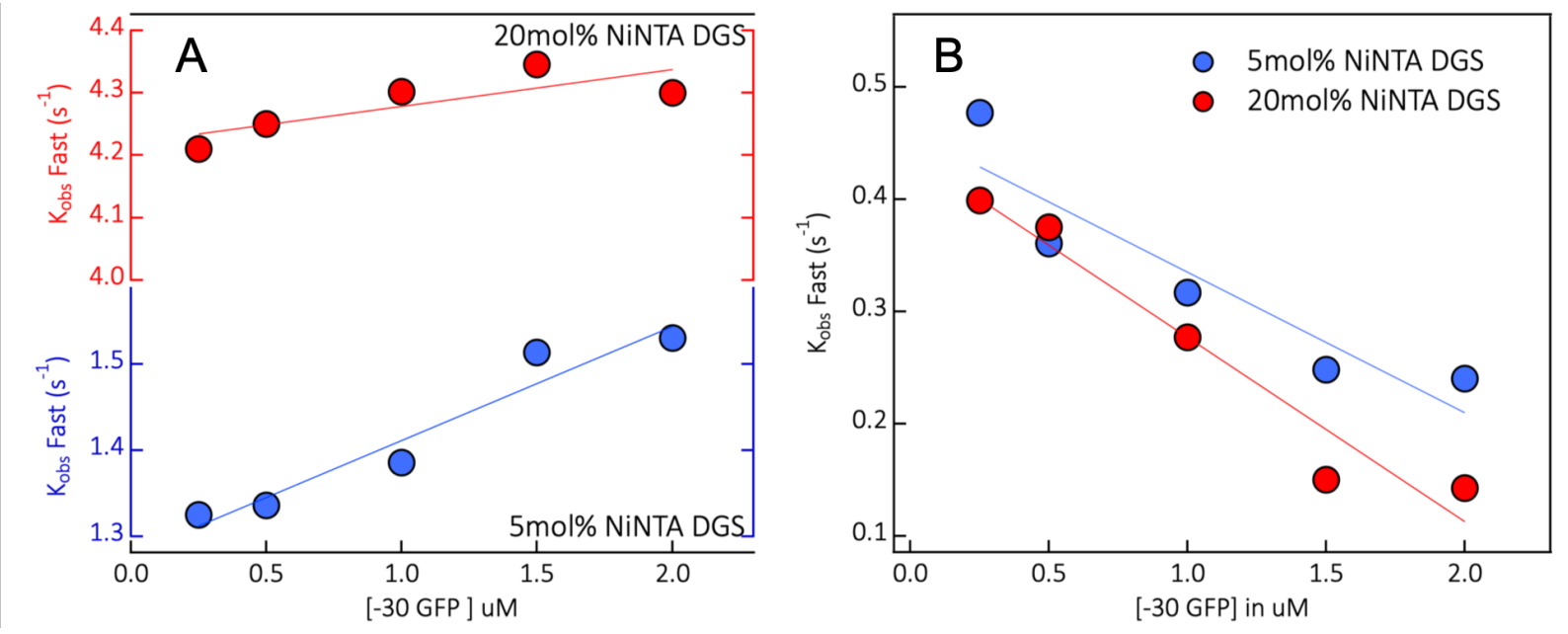

Figure S12. Effects of changing protein surface charge on binding kinetics. Observed rate constants from bi-exponential fits at high protein concentrations ( $>250 \mathrm{nM}$ ) by using $-30 \mathrm{GFP}$. (A) Fast phase rate constants at $5 \mathrm{~mol} \%$ Ni-NTA DGS (bottom) and $20 \mathrm{~mol} \% \mathrm{Ni}$-NTA DGS (top) and (B) Slow phase rate constants at $5 \mathrm{~mol} \%$ Ni-NTA DGS (bottom) and 20 mol\% Ni-NTA DGS (top). Despite changes in overall binding rates, we observed a clear trend of decreasing slow phase rate constants. 


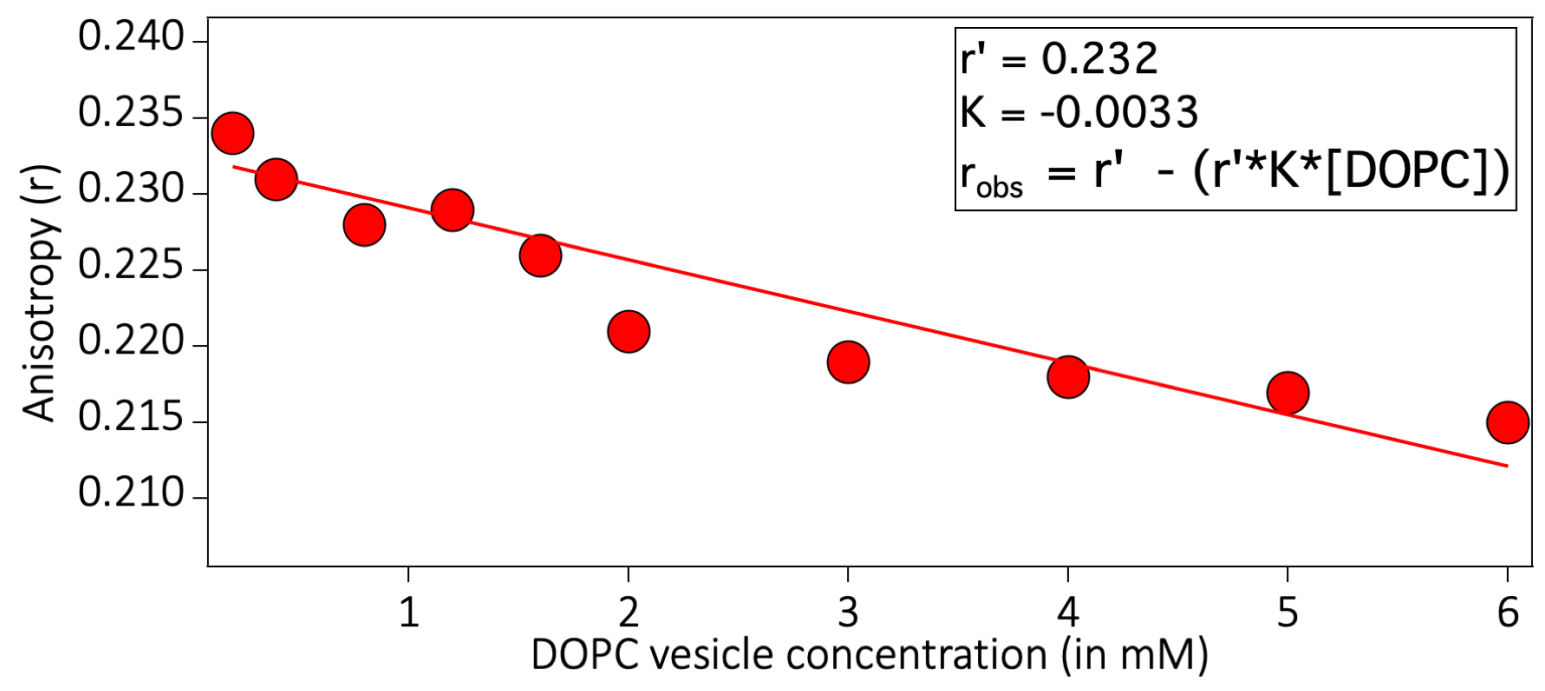

Figure S13. Anisotropy correction for turbid media. Anisotropy $(r)$ of SfGFP fluorescence in presence of liposomes void of chelator lipid. By using Ni-free liposomes, we were able to account for the increase in turbidity of the optical medium without any major changes to the physical environment of the fluorophore. Figure legend denotes the parameters used for anisotropy correction. $\mathrm{K}$ is the proportionality constant, $r$ ' is the extrapolated anisotropy at zero optical density and $r_{o b s}$ is the observed anisotropy. 

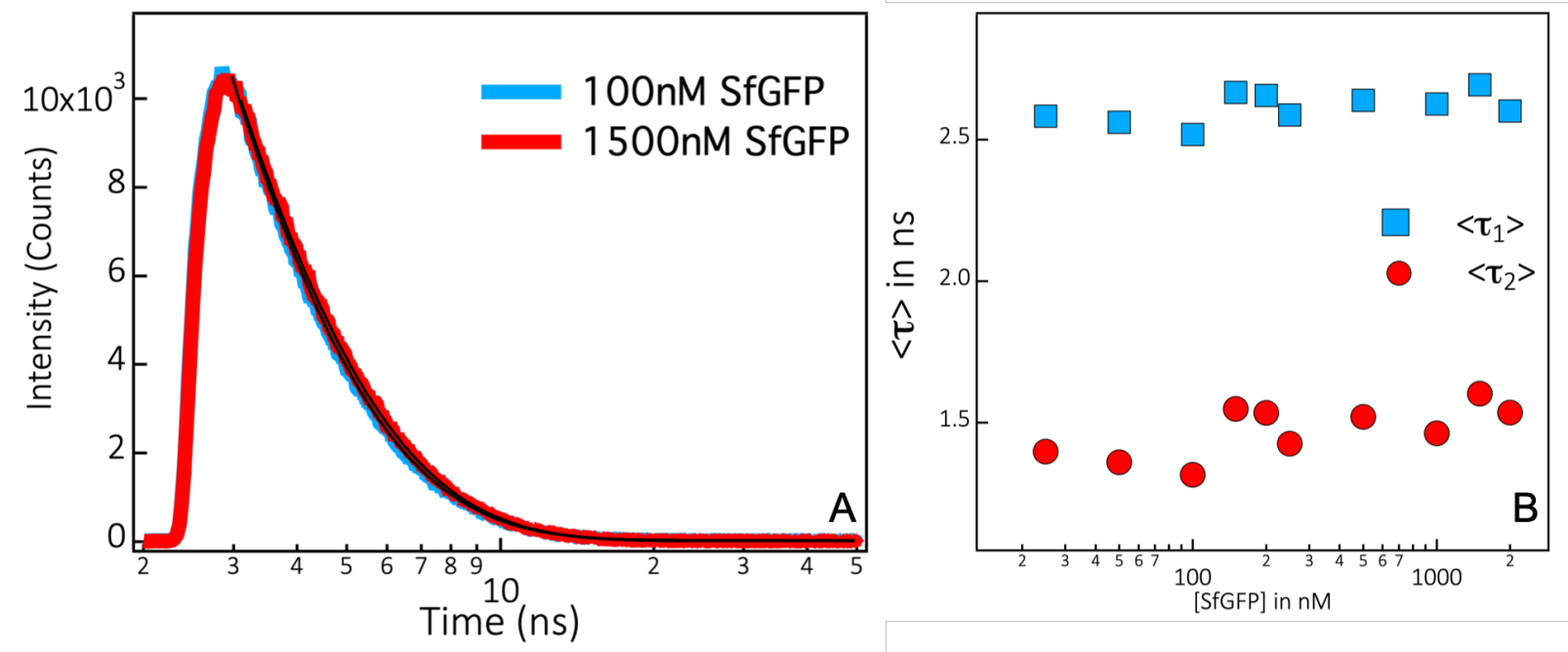

Figure S14. Fluorescence lifetime of SfGFP does not change as a result of homo-FRET. Fluorescence lifetimes $(\tau)$ acquired via time-correlated single photon counting measurements on emission signals from SfGFP-liposome complexes. (A) Representative fluorescence lifetime transients and their associated biexponential tail-fits plotted as a function of time. (B) Concentration dependence of fluorescence lifetimes of SfGFP emission plotted as a function of SfGFP concentration. Clearly, no change in fluorescence lifetime is observed irrespective of the protein concentration used. 

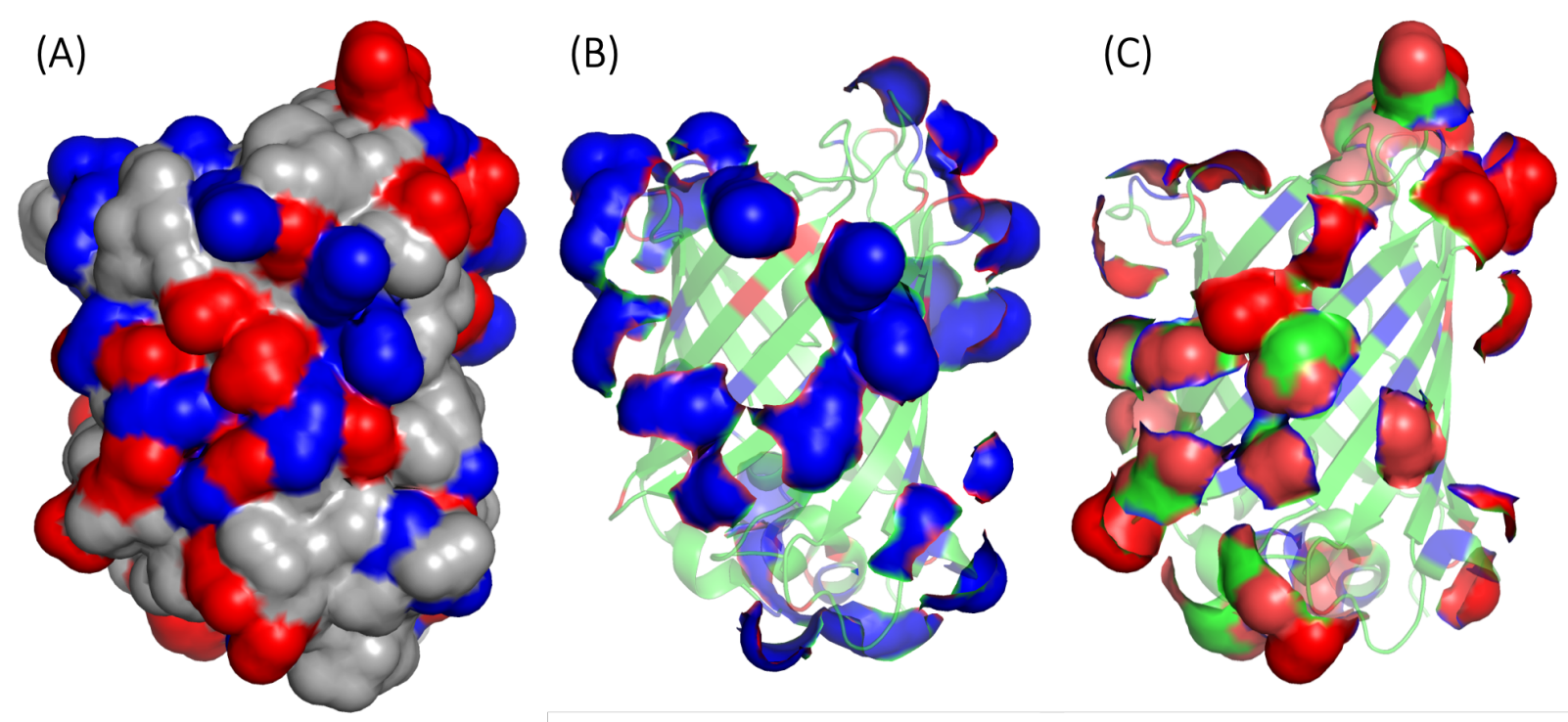

Figure S15: Charge distribution on the surface of SfGFP from analysis of the crystal structure (PDB ID: 2B3P). (A) Solvent accessible regions of the protein structure are displayed. The regions colored in red indicate negatively charged residues and regions colored in blue indicate positively charged residues. Uncharged residues are represented by gray. Non-uniform distribution of the positively charged amino acids (B) and negatively charged amino acids (C) are observed in the structure indicating the possibility of electrostatic stabilization of the side-on orientation. 


\section{Supplemental References:}

1. Reddish, M. J.; Vaughn, M. B.; Fu, R.; Dyer, R. B., Ligand-Dependent Conformational Dynamics of Dihydrofolate Reductase. Biochemistry 2016, 55, 1485-93.

2. Kozlowski, R.; Ragupathi, A.; Dyer, R. B., Characterizing the Surface Coverage of Protein-Gold Nanoparticle Bioconjugates. Bioconjug Chem 2018, 29 (8), 2691-2700.

3. Kim, H.; Afsari, H. S.; Cho, W., High-throughput fluorescence assay for membraneprotein interaction. J Lipid Res 2013, 54, 3531-8.

4. $\quad$ Cho, W.; Bittova, L.; Stahelin, R. V., Membrane binding assays for peripheral proteins. Anal Biochem 2001, 296, 153-61.

5. Reimhult, E.; Kasemo, B.; Hook, F., Rupture pathway of phosphatidylcholine liposomes on silicon dioxide. Int J Mol Sci 2009, 10, 1683-96.

6. Shnek, D. R.; Pack, D. W.; Sasaki, D. Y.; Arnold, F. H., Specific Protein Attachment to Artificial Membranes Via Coordination to Lipid-Bound Copper(II). Langmuir 1994, 10, 23822388.

7. Wegner, S. V.; Spatz, J. P., Cobalt(III) as a Stable and Inert Mediator lon between NTA and His6-Tagged Proteins. Angew Chem Int Edit 2013, 52, 7593-7596.

8. $\quad$ Chang, J.; Kim, S. A.; Lu, X.; Su, Z.; Kim, S. K.; Shin, Y. K., Fusion step-specific influence of cholesterol on SNARE-mediated membrane fusion. Biophys $J$ 2009, 96, 1839-46.

9. Lentz, B. R.; Moore, B. M.; Barrow, D. A., Light-scattering effects in the measurement of membrane microviscosity with diphenylhexatriene. Biophys J 1979, 25, 489-94. 